Data Repository 2016062

\title{
Key role of continental margin sediments in the oceanic mass balance of $\mathrm{Zn}$ and $\mathrm{Zn}$ isotopes
}

\author{
Little et al.
}

Table DR1. Summary of data and data sources reported in Figure 1

\begin{tabular}{|c|c|c|c|c|c|}
\hline & $\begin{array}{l}\text { Mean } \\
\delta^{66} \mathrm{Zn}\end{array}$ & 1SD & $\begin{array}{l}\text { Range } \\
\text { (min, } \\
\max \text { ) }\end{array}$ & $\mathrm{n}$ & Reference sources \\
\hline Lithogenic & 0.27 & 0.07 & - & 50 & $\begin{array}{l}\text { Maréchal et al. (2000); Sivry et al. (2008); Viers et al. } \\
\text { (2007); Chen et al. (2009, 2014); Sonke et al. (2008); Dong } \\
\text { et al. (2013); Little et al. (2014); Thapalia et al. (2014) }\end{array}$ \\
\hline Seawater (deep) & 0.49 & 0.07 & - & 116 & $\begin{array}{l}\text { Bermin et al. (2006); Boyle et al., 2012; Zhao et al. (2014); } \\
\text { Conway et al. (2014), Conway and John (2015) }\end{array}$ \\
\hline Rivers & 0.33 & - & $\begin{array}{l}0.21 \\
0.58\end{array}$ & 32 & $\begin{array}{l}\text { Discharge, [Zn]-weighted mean of data in Little et al. } \\
\text { (2014) }\end{array}$ \\
\hline Aeolian dust & 0.32 & 0.14 & $\begin{array}{l}0.08 \\
0.54\end{array}$ & 30 & $\begin{array}{l}\text { Maréchal et al. (2000); Dong et al. (2013); Little et al. } \\
\text { (2014) }\end{array}$ \\
\hline Hydrothermal fluids & - & - & $\begin{array}{l}-0.5 \\
0.24\end{array}$ & 21 & $\begin{array}{l}\text { End-member estimate of Conway and John (2015) and } \\
\text { [Zn]-weighted mean of data in John et al. (2008) }\end{array}$ \\
\hline Fe-Mn sediments & 0.96 & 0.15 & $\begin{array}{l}0.53 \\
1.23\end{array}$ & 69 & Maréchal et al. (2000); Little et al. (2014) \\
\hline Biogenic carbonate & 1.07 & 0.14 & $\begin{array}{l}0.81 \\
1.34\end{array}$ & 26 & Pichat et al. (2003) \\
\hline Opal & 1.03 & 0.19 & $\begin{array}{l}0.71 \\
1.47\end{array}$ & 20 & Andersen et al. (2011) \\
\hline \multirow[t]{2}{*}{ Margin sediments } & 0.32 & 0.03 & - & 10 & Peru margin: This study \\
\hline & 0.08 & 0.06 & - & 26 & Mexican and California margins: This study \\
\hline
\end{tabular}


Figure DR1. Site locations plotted superimposed on average sea surface $\mathrm{PO}_{4}$ concentrations $\left(\mu \mathrm{mol} \mathrm{kg}{ }^{-1}\right)$. Phosphate data from the 2009 World Ocean Atlas (Garcia et al., 2010). Image plotted using ODV software (Schlitzer, R., Ocean Data View, odv.awi.de, 2015)

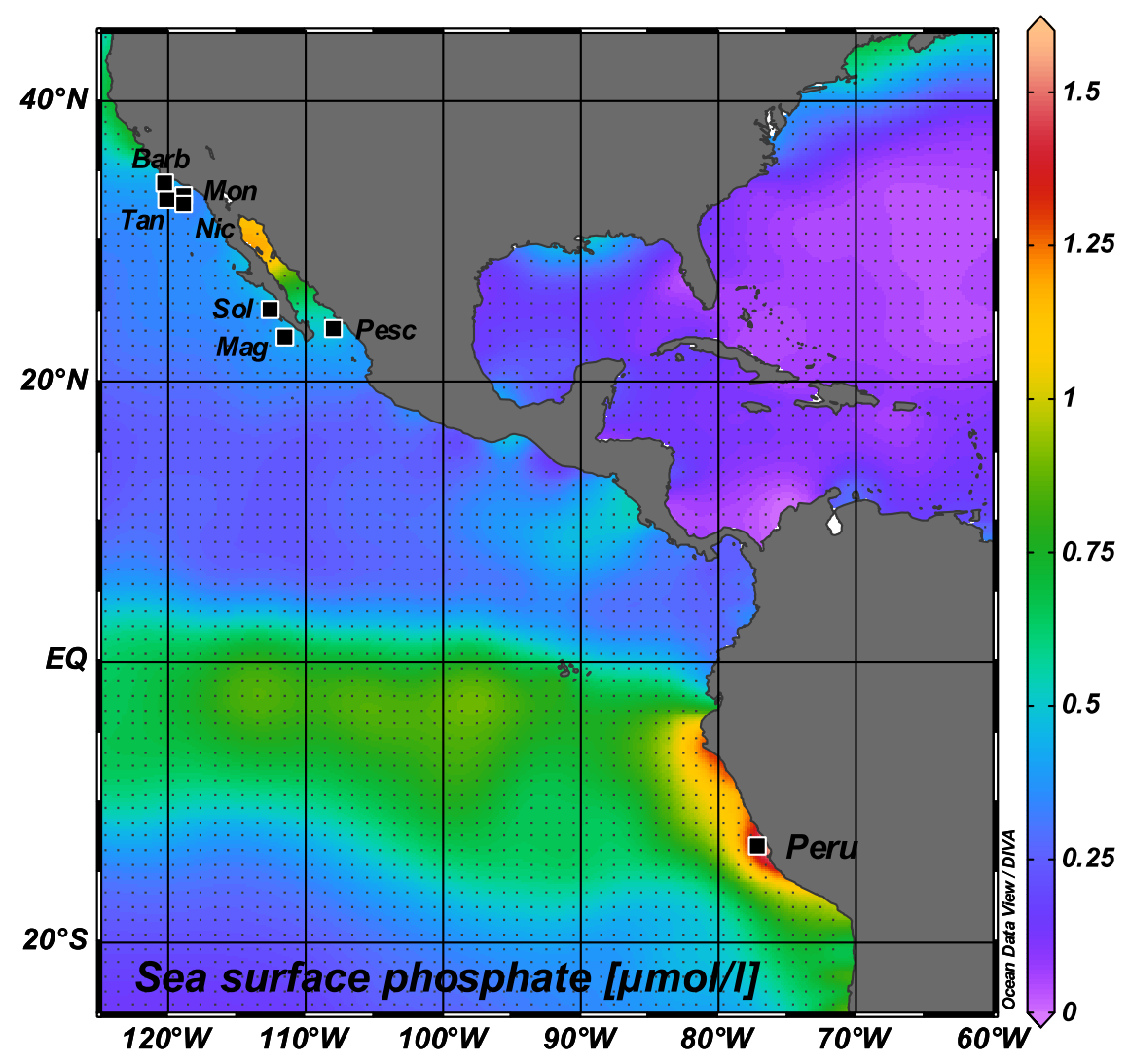


Table DR2. Results.

\begin{tabular}{|c|c|c|c|c|c|c|c|c|c|c|c|}
\hline Site & $\begin{array}{l}\text { Depth } \\
\mathrm{cm}\end{array}$ & $\begin{array}{c}\mathrm{C}_{\text {org }} \\
\mathrm{wt} \%\end{array}$ & $\begin{array}{c}\text { Total S } \\
\text { wt } \%\end{array}$ & $\begin{array}{c}\mathrm{Al} \\
\mathrm{wt} \%\end{array}$ & $\begin{array}{c}\mathrm{Zn} / \mathrm{Al} \\
\left(\times 10^{-4}\right)\end{array}$ & $\mathrm{Zn}_{\mathrm{XS}}$ & $\delta^{66} \mathrm{Zn}_{\text {bulk }}$ & $\mathrm{X}_{\text {lith }}$ & $2 \mathrm{SD}$ & $\delta^{66} \mathrm{Zn}_{\text {auth }}$ & $2 \mathrm{SD}$ \\
\hline \multirow[t]{3}{*}{ Santa Barbara } & 1.3 & 3.01 & 0.53 & 6.63 & 15.5 & 47.3 & 0.11 & 0.54 & 0.13 & -0.10 & 0.25 \\
\hline & 8.3 & 1.50 & 0.62 & 5.97 & 15.3 & 41.5 & 0.05 & 0.55 & 0.13 & -0.23 & 0.28 \\
\hline & 41 & 2.93 & 0.68 & 6.32 & 15.1 & 42.3 & 0.12 & 0.56 & 0.13 & -0.07 & 0.26 \\
\hline \multirow{4}{*}{ Santa Monica } & 1.5 & 5.73 & 0.48 & 5.39 & 24.7 & 87.6 & 0.10 & 0.34 & 0.08 & 0.02 & 0.12 \\
\hline & 3.5 & 5.23 & 0.23 & 6.21 & 18.7 & 64.0 & 0.14 & 0.45 & 0.11 & 0.03 & 0.17 \\
\hline & 7.5 & 4.19 & 0.28 & 6.17 & 17.8 & 58.6 & 0.12 & 0.47 & 0.11 & -0.01 & 0.18 \\
\hline & 20 & 4.50 & 0.31 & 6.14 & 18.2 & 60.1 & 0.15 & 0.46 & 0.11 & 0.05 & 0.18 \\
\hline \multirow[t]{4}{*}{ San Nicolas } & 2.8 & 4.92 & 0.41 & 4.81 & 28.7 & 97.7 & 0.14 & 0.29 & 0.07 & 0.09 & 0.11 \\
\hline & 4.3 & 4.85 & 0.40 & 5.13 & 28.7 & 104.0 & 0.14 & 0.29 & 0.07 & 0.08 & 0.11 \\
\hline & 14.5 & 4.25 & nd & 4.75 & 20.3 & 56.5 & 0.14 & 0.41 & 0.10 & 0.04 & 0.15 \\
\hline & 33 & 4.41 & 0.44 & 5.11 & 34.8 & 135.0 & 0.15 & 0.24 & 0.06 & 0.12 & 0.09 \\
\hline \multirow[t]{4}{*}{ Tanner Basin } & 1.5 & 6.69 & 0.33 & 3.29 & 38.3 & 98.5 & 0.10 & 0.22 & 0.05 & 0.05 & 0.09 \\
\hline & 3.5 & 6.35 & 0.36 & 3.33 & 39.3 & 103.2 & 0.06 & 0.21 & 0.05 & 0.01 & 0.09 \\
\hline & 7.5 & 5.96 & 0.35 & 2.96 & 36.3 & 82.5 & 0.02 & 0.23 & 0.06 & -0.06 & 0.09 \\
\hline & 13.5 & 4.31 & 0.16 & 3.75 & 28.1 & 73.8 & 0.08 & 0.30 & 0.07 & 0.00 & 0.11 \\
\hline \multirow[t]{4}{*}{ Pescadero } & 2.5 & 3.55 & 0.49 & 6.47 & 14.4 & 39.2 & 0.02 & 0.58 & 0.14 & -0.35 & 0.35 \\
\hline & 11.5 & 3.45 & 0.44 & 7.05 & 14.7 & 44.3 & -0.05 & 0.57 & 0.14 & -0.51 & 0.37 \\
\hline & 23 & 3.07 & 0.38 & 7.35 & 15.0 & 48.3 & -0.02 & 0.56 & 0.13 & -0.40 & 0.33 \\
\hline & 31 & 3.62 & 0.58 & 6.92 & 14.6 & 43.2 & 0.02 & 0.57 & 0.14 & -0.34 & 0.33 \\
\hline \multirow{4}{*}{ Soledad } & 1.5 & 6.47 & 0.54 & 4.12 & 15.7 & 29.9 & 0.01 & 0.54 & 0.13 & -0.31 & 0.28 \\
\hline & 11.5 & 6.39 & 0.59 & 4.39 & 15.1 & 29.3 & 0.00 & 0.56 & 0.13 & -0.36 & 0.31 \\
\hline & 21 & 6.66 & 0.78 & 4.13 & 16.3 & 32.6 & 0.03 & 0.52 & 0.12 & -0.24 & 0.25 \\
\hline & 31 & 6.23 & 0.61 & 4.53 & 15.1 & 30.6 & 0.05 & 0.56 & 0.13 & -0.23 & 0.28 \\
\hline \multirow[t]{3}{*}{ Magdalena } & 0.5 & 10.03 & 0.85 & 2.46 & 41.3 & 81.2 & 0.11 & 0.20 & 0.05 & 0.07 & 0.08 \\
\hline & 4.5 & 11.71 & 0.64 & 2.50 & 45.9 & 93.7 & 0.12 & 0.18 & 0.04 & 0.09 & 0.08 \\
\hline & 6.5 & 12.68 & 0.66 & 2.41 & 46.6 & 92.2 & 0.13 & 0.18 & 0.04 & 0.10 & 0.08 \\
\hline \multirow{10}{*}{ Peru } & 0.5 & 15.50 & 1.38 & 2.68 & 35.5 & 72.7 & 0.34 & 0.24 & 0.06 & 0.36 & 0.09 \\
\hline & 1.0 & 16.00 & 1.37 & 2.83 & 32.1 & 67.1 & 0.33 & 0.26 & 0.06 & 0.34 & 0.10 \\
\hline & 1.7 & 15.70 & 1.44 & 2.87 & 32.2 & 68.1 & 0.28 & 0.26 & 0.06 & 0.29 & 0.10 \\
\hline & 2.4 & 15.40 & 1.54 & 3.01 & 30.5 & 66.6 & 0.32 & 0.28 & 0.07 & 0.34 & 0.10 \\
\hline & 4.8 & 14.50 & 1.45 & 2.92 & 31.2 & 66.5 & 0.36 & 0.27 & 0.06 & 0.39 & 0.10 \\
\hline & 8.2 & 12.80 & 1.35 & 2.61 & 30.6 & 58.1 & 0.34 & 0.27 & 0.07 & 0.36 & 0.10 \\
\hline & 15.0 & 11.70 & 1.18 & 2.53 & 26.6 & 46.1 & 0.28 & 0.32 & 0.08 & 0.29 & 0.11 \\
\hline & 15.7 & 12.10 & 1.14 & 2.85 & 24.2 & 45.2 & 0.32 & 0.35 & 0.08 & 0.34 & 0.12 \\
\hline & 18.4 & 14.40 & 1.30 & 3.61 & 23.1 & 53.2 & 0.29 & 0.36 & 0.09 & 0.30 & 0.13 \\
\hline & 19.1 & 14.80 & 1.29 & 3.56 & 24.6 & 57.9 & 0.36 & 0.34 & 0.08 & 0.41 & 0.12 \\
\hline \multicolumn{12}{|l|}{ Site averages } \\
\hline Santa Bar & - & 2.48 & 0.61 & 6.31 & 15.3 & 43.7 & 0.09 & 0.55 & 0.13 & -0.13 & 0.26 \\
\hline Santa Monica & - & 4.91 & 0.32 & 5.98 & 19.9 & 67.6 & 0.13 & 0.43 & 0.10 & 0.02 & 0.16 \\
\hline San Nicolas & - & 4.61 & 0.41 & 4.95 & 28.1 & 98.3 & 0.14 & 0.30 & 0.07 & 0.09 & 0.11 \\
\hline Tanner Basin & - & 5.83 & 0.30 & 3.33 & 35.5 & 89.5 & 0.07 & 0.24 & 0.06 & 0.00 & 0.09 \\
\hline Pescadero & - & 3.42 & 0.47 & 6.95 & 14.7 & 43.7 & -0.01 & 0.57 & 0.14 & -0.40 & 0.34 \\
\hline Soledad & - & 6.44 & 0.63 & 4.29 & 15.5 & 30.6 & 0.02 & 0.54 & 0.13 & -0.29 & 0.28 \\
\hline Magdalena & - & 11.47 & 0.72 & 2.46 & 44.6 & 89.0 & 0.12 & 0.19 & 0.04 & 0.08 & 0.08 \\
\hline Peru & - & 14.29 & nd & 2.95 & 29.1 & 60.2 & 0.32 & 0.29 & 0.07 & 0.34 & 0.10 \\
\hline
\end{tabular}

nd: not done. 
Figure DR2. Uncertainty on calculated $\delta^{66} \mathrm{Zn}_{\text {auth }}$ values (represented as calculated 2SD) increases as a function of the lithogenic fraction in the sediment (calculated $\mathrm{X}_{\text {lith }}$ ). See text for details of calculations.

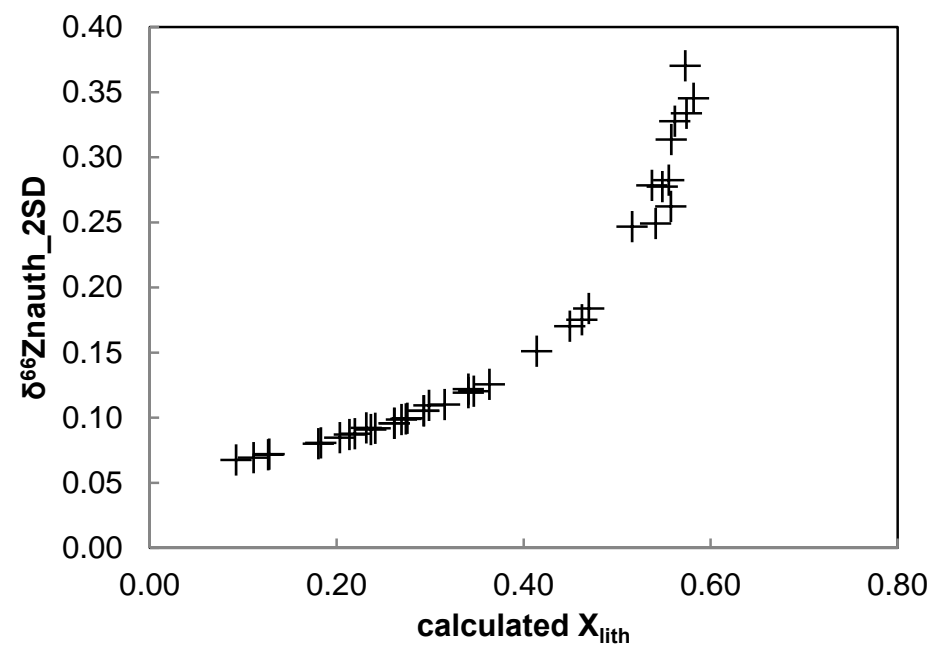

Figure DR3. A secondary effect on the uncertainty on calculated $\delta^{66} \mathrm{Zn}_{\text {auth }}$ values is the initial difference between $\delta^{66} \mathrm{Zn}_{\text {lith }}$ and $\delta^{66} \mathrm{Zn}_{\text {bulk }}$. The greater this difference, the larger the uncertainty on $\delta^{66} \mathrm{Zn}_{\text {auth }}$ values.

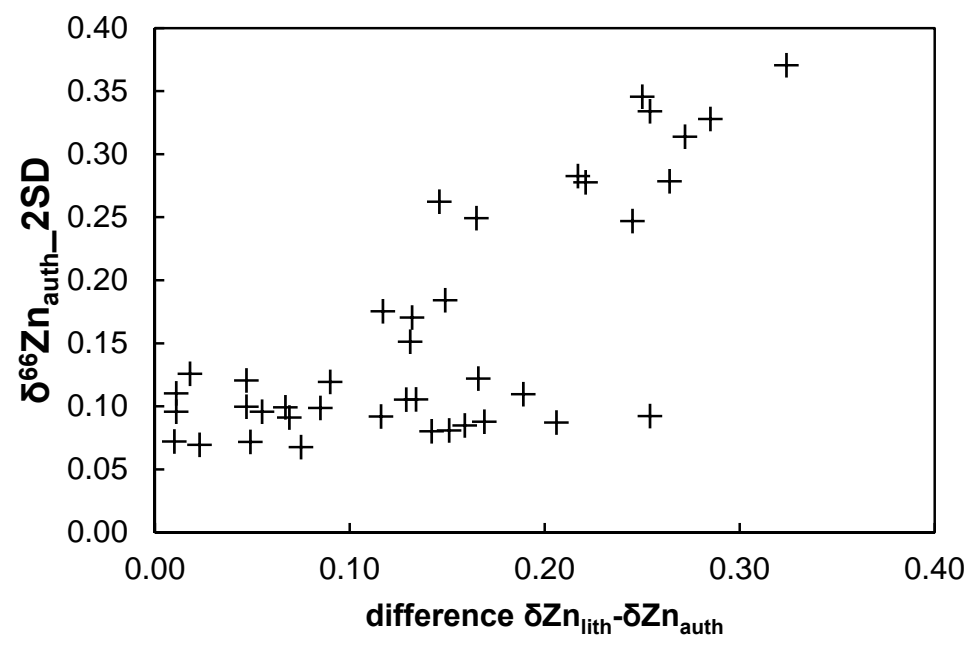




\section{$\underline{\text { References }}$}

Andersen, M. B., Vance, D., Archer, C., Anderson, R. F., Ellwood, M. J., and Allen, C. S., 2011, The $\mathrm{Zn}$ abundance and isotopic composition of diatom frustules, a proxy for $\mathrm{Zn}$ availability in ocean surface seawater: Earth and Planetary Science Letters, v. 301, no. 1, p. 137-145.

Bermin, J., Vance, D., Archer, C., and Statham, P. J., 2006, The determination of the isotopic composition of $\mathrm{Cu}$ and $\mathrm{Zn}$ in seawater: Chemical Geology, v. 226, no. 3, p. 280-297.

Böning, P., Brumsack, H. J., Böttcher, M. E., Schnetger, B., Kriete, C., Kallmeyer, J., and Borchers, S. L., 2004, Geochemistry of Peruvian near-surface sediments: Geochimica et Cosmochimica Acta, v. 68, no. 21, p. 4429-4451.

Chen, J., Gaillardet, J., Louvat, P., and Huon, S., 2009, Zn isotopes in the suspended load of the Seine River, France: Isotopic variations and source determination: Geochimica et Cosmochimica Acta, v. 73, no. 14, p. 4060-4076.

Chen, J. B., Gaillardet, J., Dessert, C., Villemant, B., Louvat, P., Crispi, O. et al., 2014, Zn isotope compositions of the thermal spring waters of La Soufrière volcano, Guadeloupe Island: Geochimica et Cosmochimica Acta, v. 127, p. 67-82.

Conway, T. M., and John, S. G., 2014, The biogeochemical cycling of zinc and zinc isotopes in the North Atlantic Ocean: Global Biogeochemical Cycles, v. 28, no. 10, p. 1111-1128.

Conway, T. M., and John, S. G., 2015, The cycling of iron, zinc and cadmium in the North East Pacific Ocean-insights from stable isotopes: Geochimica et Cosmochimica Acta, v. 164, p. $262-283$.

Dong, S., Weiss, D. J., Strekopytov, S., Kreissig, K., Sun, Y., Baker, A. R., and Formenti, P., 2013, Stable isotope ratio measurements of $\mathrm{Cu}$ and $\mathrm{Zn}$ in mineral dust (bulk and size fractions) from the Taklimakan Desert and the Sahel and in aerosols from the eastern tropical North Atlantic Ocean: Talanta, v. 114, p. 103-109.

Garcia, H. E., Locarnini, R.A., Boyer, T. P., Antonov, J. I., Zweng, M. M., Baranova, O. K., and Johnson, D. R., 2010, World Ocean Atlas 2009, Volume 4: Nutrients (phosphate, nitrate, silicate). S. Levitus, Ed. NOAA Atlas NESDIS 71, U.S. Government Printing Office, Washington, D.C., 398 p.

John, S. G., Rouxel, O. J., Craddock, P. R., Engwall, A. M., and Boyle, E. A., 2008, Zinc stable isotopes in seafloor hydrothermal vent fluids and chimneys: Earth and Planetary Science Letters, v. 269, no. 1, p. 17-28.

Little, S. H., Vance, D., Walker-Brown, C., and Landing, W. M., 2014, The oceanic mass balance of copper and zinc isotopes, investigated by analysis of their inputs, and outputs to ferromanganese oxide sediments: Geochimica et Cosmochimica Acta, v. 125, p. 673-693.

Pichat, S., Douchet, C., and Albarède, F., 2003, Zinc isotope variations in deep-sea carbonates from the eastern equatorial Pacific over the last 175 ka: Earth and Planetary Science Letters, v. 210, no. 1, p. 167-178. 
Maréchal, C. N., Nicolas, E., Douchet, C., and Albarède, F., 2000, Abundance of zinc isotopes as a marine biogeochemical tracer: Geochemistry, Geophysics, Geosystems, v. 1, no. 5.

Rudnick, R. L., and Gao, S., 2003, Composition of the continental crust: Treatise on geochemistry, v. 3, p. 1-64.

Sivry, Y., Riotte, J., Sonke, J. E., Audry, S., Schäfer, J., Viers, J. et al., 2008, Zn isotopes as tracers of anthropogenic pollution from Zn-ore smelters, The Riou Mort-Lot River system: Chemical Geology, v. 255, no. 3, p. 295-304.

Sonke, J. E., Sivry, Y., Viers, J., Freydier, R., Dejonghe, L., André, L. et al., 2008, Historical variations in the isotopic composition of atmospheric zinc deposition from a zinc smelter: Chemical Geology, v. 252, no. 3, p. 145-157.

Thapalia, A., Borrok, D. M., Van Metre, P. C., and Wilson, J., 2014, Zinc Isotopic Signatures in Eight Lake Sediment Cores from Across the United States: Environmental science \& technology, v. 49, no. 1, p. 132-140.

Viers, J., Oliva, P., Nonell, A., Gélabert, A., Sonke, J. E., Freydier, R. et al., 2007, Evidence of $\mathrm{Zn}$ isotopic fractionation in a soil-plant system of a pristine tropical watershed (Nsimi, Cameroon): Chemical Geology, v. 239, no. 1, p. 124-137.

Zhao, Y., Vance, D., Abouchami, W., and De Baar, H. J. W., 2014, Biogeochemical cycling of zinc and its isotopes in the Southern Ocean: Geochimica et Cosmochimica Acta, v. 125, p. 653-672. 